\title{
Ferroelectric Liquid-Crystalline Polytartrate
}

\author{
Seiji UjIIE and Kazuyoshi IImURA \\ Department of Chemistry, Faculty of Science, Science University of Tokyo, \\ Kagurazaka, Shinjuku-ku, Tokyo 162, Japan
}

(Received April 6, 1991)

\begin{abstract}
Ferroelectric liquid-crystalline polytartrate (LCPTT) was prepared by melt polycondensation, and studied for thermal properties and ferroelectric behavior. Cholesteric and chiral smectic $\mathrm{C}^{*}\left(\mathrm{SmC}^{*}\right)$ phases were observed. LCPTT exhibited the large spontaneous polarization value of $5 \mathrm{mC} \mathrm{m}^{-2}$ in the $\mathrm{SmC}^{*}$ phase because of a stereopolar coupling of the chiral tartrate moiety.

KEY WORDS Ferroelectric / Liquid-Crystalline Polytartrate / Chiral Smectic C* Phase / Thermal Properties / Spontaneous Polarization / Dielectric / Rochelle Salt /
\end{abstract}

Ferroelectric liquid-crystalline polymers (FLCPs) were studied on thermal and electrooptic properties from fundamental and applied viewpoints. ${ }^{1-6}$ In general, FLCPs are known to have the structure such that a chiral mesogenic side-chain is linked to acryl, methacryl or siloxane types of polymer backbone. The structure of the chiral mesogenic side-chain is similar to that of a low-mass ferroelectric liquid crystal. In this case, a chiral center is usually located in the mesogenic end group. In the case of liquid-crystalline side-chain polymers, a chiral center can be also introduced in the mesogenic unit, the spacer or polymer backbone. However, there have been few reports on those types of FLCPs. We synthesized the ferroelectric liquid-crystalline polytartrate (LCPTT) as FLCP with the chiral centers in the polymer backbone. ${ }^{8)}$ In this work, we study liquid-crystalline properties and ferroelectric behavior in LCPTT.

\section{RESULTS AND DISCUSSION}

The structure of LCPTT is shown in Figure 1. LCPTT exhibited cholesteric $(\mathrm{Ch})$ and chiral smectic $\mathrm{C}^{*}\left(\mathrm{SmC}^{*}\right)$ phases. As shown in Figure 2a, a fingerprint texture was observed in the $\mathrm{Ch}$ phase. The cholesteric pitch (P) was determined from the fingerprint texture, and its temperature dependence is shown in Figure 3. The cholesteric pitch became narrow with decreasing temperature. The minimum value of $\mathbf{P}$ for every LCPTTs was about $12-13 \mu \mathrm{m}$. At the transition point of the $\mathrm{Ch}$ to the $\mathrm{SmC}^{*}$ phase, the cholesteric pitch rapidly increased. The SmC* phase exhibited striped fan, schlierenlike and pseudo-homeotropic textures. The striped fan texture is shown in Figure 2b. The optical-rotatory power measured in the pseudohomeotropic structure was +45 degree $/ \mathrm{mm}$. The striped fan texture and optical-rotatory power obtained in LCPTT indicate that the helical structure is present in the $\mathrm{SmC}^{*}$ phase.

Phase transition temperatures of LCPTTs are listed in Table I. With increasing molecular

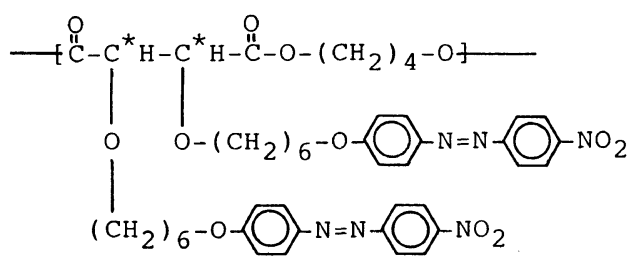

Figure 1. Structure of LCPTT. 


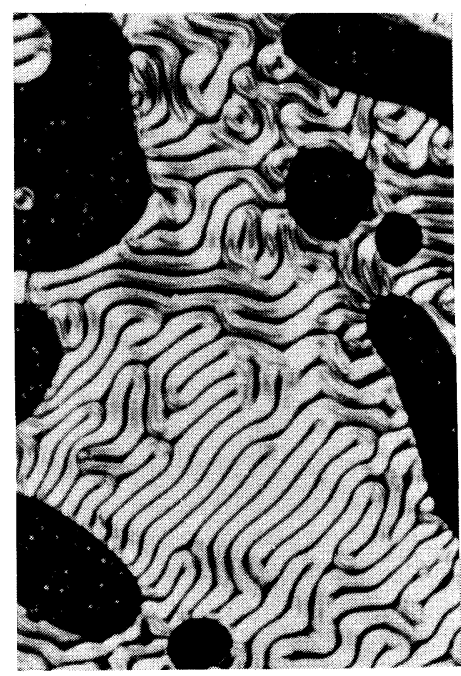

(a)

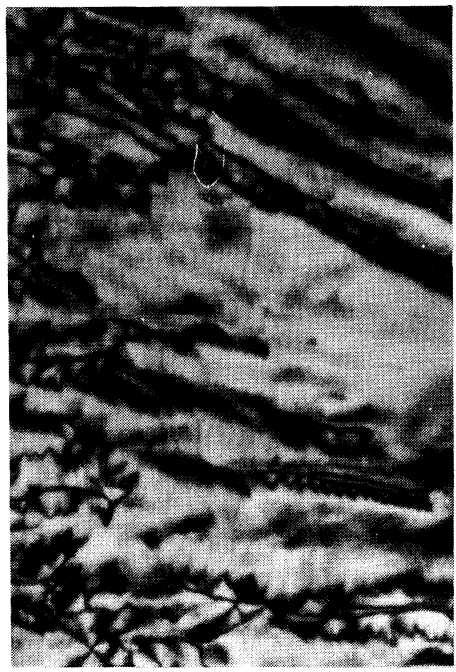

(b)

Figure 2. Optical textures of LCPTT: (a) fingerprint texture (Ch phase); (b) striped fan texture (SmC* phase). Magnification $\times 200$.

Table I. Phase transition temperatures of liquid-crystalline polytartrates

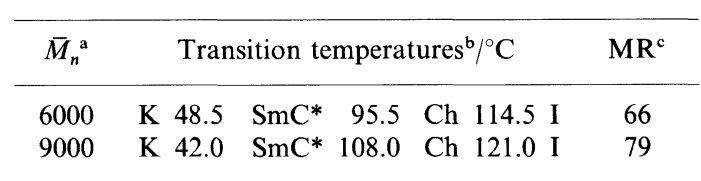

a Number average of molecular weight determined by GPC in THF, calibrated against polystyrene standards.

b $\mathrm{K}$, solid phase, $\mathrm{SmC}^{*}$, chiral smectic $\mathrm{C}^{*}$ phase; $\mathrm{Ch}$, cholesteric phase; I, isotropic phase.

c Mesomorphic temperature range.

weight, the Ch-isotropic temperature increased and the mesomorphic temperature range (MR) became broader. This corresponds to the general fact that increase of the molecular weight enhances the formation of the liquidcrystalline state. ${ }^{9,10}$

Figure 4 shows thermo-optic analysis curves of LCPTT. On cooling the transmitted light intensity (TLI) in the $\mathrm{Ch}$ phase increased because of birefringence of the fingerprint texture. In the case of the $\mathrm{SmC}^{*}$ phase, the TLI decreased owing to the formation of a pseudo-homeotropic structure. On cooling, the exothermal peak indicating crystallization

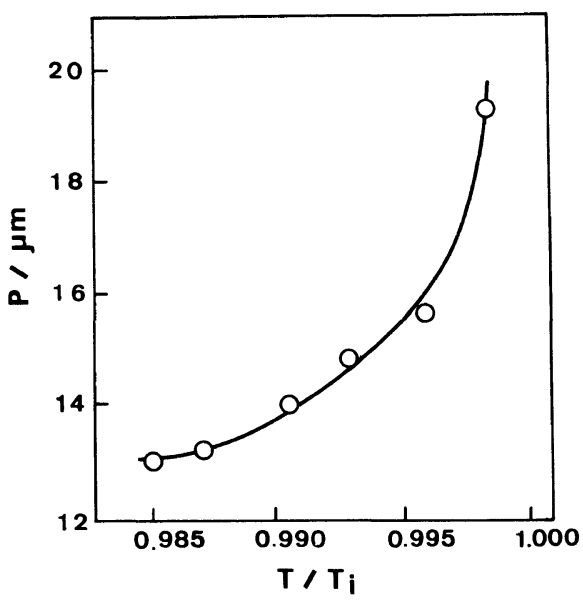

Figure 3. Temperature dependence of cholesteric pitch (p) for LCPTT $\left(\bar{M}_{n}=6000\right): T_{i}$, isotropization temperatures, $T$, measuring temperature.

at the transition temperature of the $\mathrm{SmC}^{*}$ phase to the solid state was found by DSC measurement as shown in Figure 5. In the wide angle $\mathrm{X}$-ray scattering region, only an amorphous halo was observed in the $\mathrm{SmC}^{*}$ phase. However, two sharp diffraction peaks appeared at $2 \theta /$ degree of 17 and 25 in the solid state owing to crystallization. However, TLI did not 


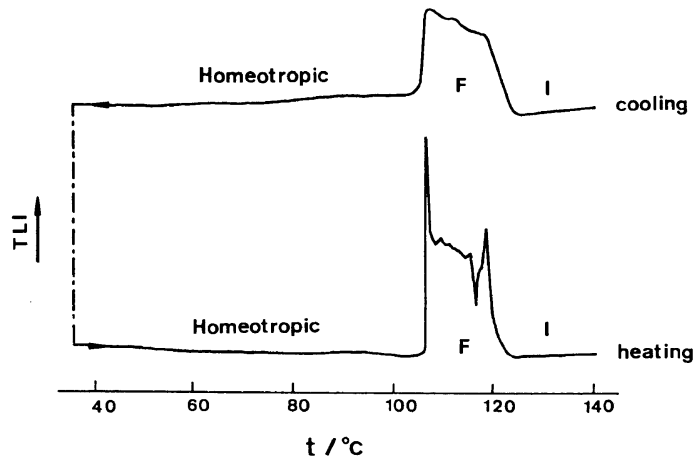

Figure 4. Thermo-optic analysis curves for LCPTT in sandwich cell without alignment treatment: I, isotropic phase; F, fingerprint texture; TLI, transmitted light intensity.

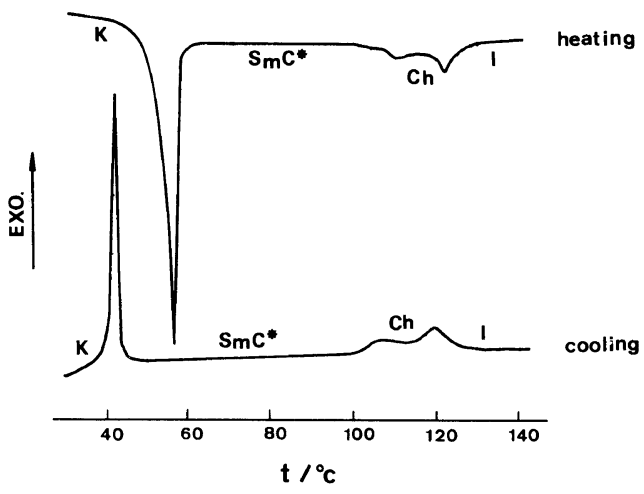

Figure 5. DSC curves of LCPTT.

change at the transition temperature of the SmC* phase to the solid state, and the pseudo-homeotropic structure remained unaltered in the solid phase. Further, the layer spacing determined from X-ray measurement was $2.94 \mathrm{~nm}$ in the $\mathrm{SmC}^{*}$ phase, and unalterable in the solid state. These results obtained by polarizing microscopic observation and X-ray measurement demonstrate that alignment of the mesogenic side-chain in the $\mathrm{SmC}^{*}$ layer is similar to that in the solid state. The temperature dependence of birefringence of LCPTT is shown in Figure 6. By rubbing treatment of the prism of the Abbe refractometer, the cholesteric helix was unwound to form a homogeneously nematic orientation, and the birefringence of liquid-crystalline phase be-

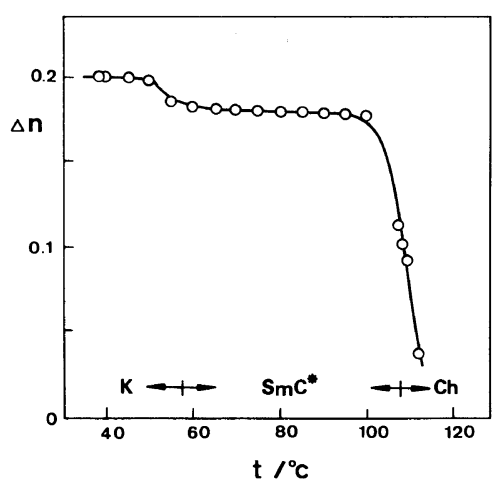

Figure 6. Temperature dependence of birefringence $(\Delta n)$ on heating.

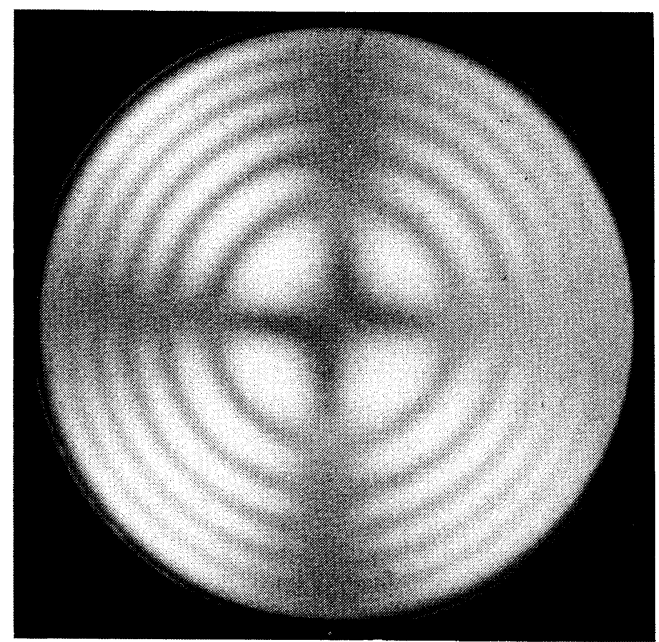

Figure 7. Conoscopic figure characteristics of optically biaxial state aligned following application of an AC electric field of LCPTT.

came positive. The birefringence in the mesophase increases with decreasing temperature, and becomes constant in the $\mathrm{SmC}^{*}$ phase. The birefringenece in the solid state increases only slightly in comparison with that in the SmC* phase. This also indicates that the orientational order of the mesogenic side-chain in the $\mathrm{SmC}^{*}$ phase practically remains unaltered in the solid state.

As shown in Figure 7, a conoscopic figure characteristic of an optically biaxial state was observed for a sample aligned on application of the AC electric field of $6 \mathrm{MV}_{\mathrm{rms}} / \mathrm{m} / 5 \mathrm{kHz} .{ }^{11}$ 


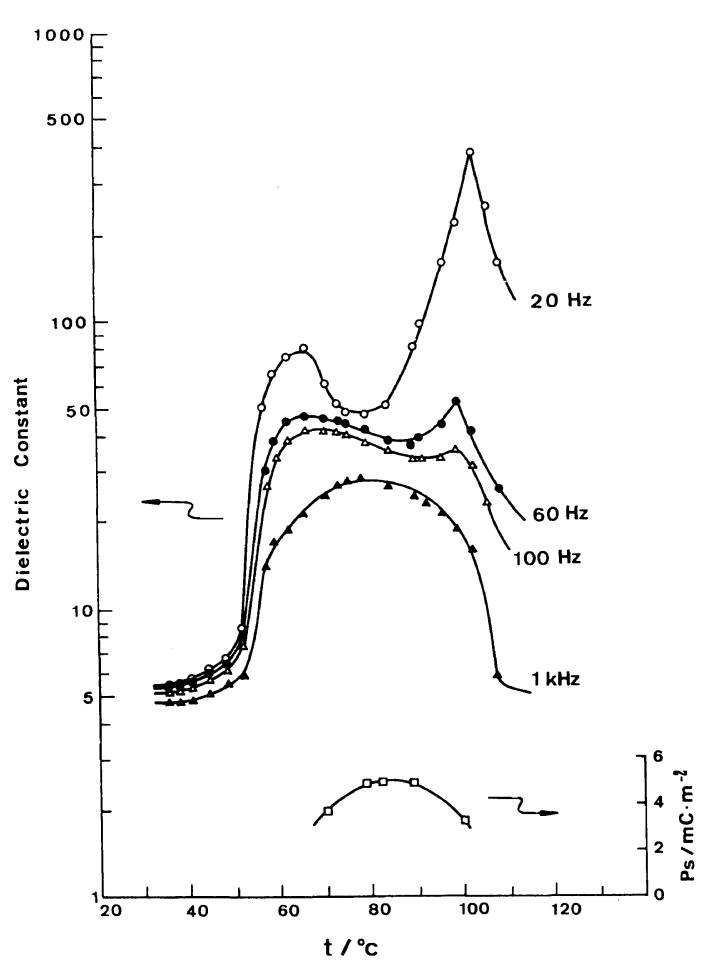

Figure 8. Temperature dependence of dielectric constant and spontaneous polarization $\left(P_{\mathrm{s}}\right)$ of LCPTT.

The orientational order parameter of this optically biaxial sample was 0.95 . The orientational order parameter was estimated from the half-width of the X-ray scattering peak measured by rotating the aligned sample in the $\mathrm{X}-\mathrm{Y}$ plane around the $\mathrm{X}$-axis (the $\mathrm{X}$-ray beam direction). ${ }^{12}$

The dielectric constant as a function of temperature is shown in Figure 8. The dielectric constant decreases with increasing frequency. The maximum dielectric constants appear at the temperatures near the solid-SmC* and the $\mathrm{SmC}^{*}$-Ch phase transition points. Similar data were also found for the Rochelle salt (potassium sodium tartrate tetrahydride) prepared from tartaric acid. ${ }^{13}$ These dielectric peaks indicate the existence of paraelectricferroelectric transition. This is explained by the Curie-Weiss' law, and solid-SmC* and $\mathrm{SmC}^{*}$-Ch phase transition points correspond to the Curie temperature.

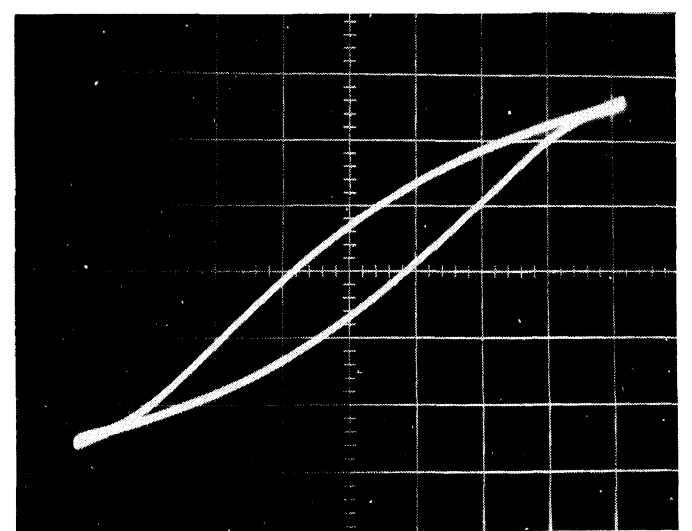

Figure 9. Hysteresis loop observed in $\mathrm{SmC}^{*}$ phase $\left(80^{\circ} \mathrm{C}\right.$, $10 \mathrm{~Hz}$ ): Horizontal axis, $500 \mathrm{kV} \mathrm{m}^{-1} / \mathrm{div}$; vertical axis, $4.6 \mathrm{mC} \mathrm{m}^{-2} / \mathrm{div}$.

A hysteresis loop observed in the $\mathrm{SmC}^{*}$ phase $\left(80^{\circ} \mathrm{C}\right)$ of LCPTT is shown in Figure 9. The value of the spontaneous polarization $\left(P_{\mathrm{s}}\right)$ is shown in Figure 8. LCPTT exhibits a large $P_{\mathrm{s}}$ value of $5 \mathrm{mC} \mathrm{m}^{-2} . P_{\mathrm{s}}$ of ferroelectric liquid-crystalline polymethacrylates, polyacrylates and polysiloxanes with chiral center in the mesogenic terminal group have been reported by several research groups. $P_{\mathrm{s}}$ of LCPTT studied in our work is 3.3 to 500 times as large as their $P_{\mathrm{s}}$ value. $^{1-6} \quad P_{\mathrm{s}}$ is well-known to become small when a chiral center is located in a nonpolar group, and is far apart from a mesogenic group. The chiral centers in the tartrate unit are separated with the hexamethylene spacer from the mesogenic group. However, the polar ether oxygens and carbonyl groups are directly attached to two chiral centers $\left(\mathrm{C}^{*}\right.$ and $\left.\mathrm{C}^{*}\right)$ in the tartrate unit. In addition, the Stuart type of the space-filling molecular model for the tartrate unit indicated that the internal rotation around the $\mathrm{C} 2{ }^{*}-\mathrm{C} 3 *$ bond in the tartrate unit is very restricted because of steric hindrance. In this case, stereopolar coupling takes place, and the tartrate unit structurally forms a large polarization. Furthermore, the molecular motion of the tartrate unit locating in the skeletal main chain is restricted in comparison with that 


$$
\begin{gathered}
\mathrm{C}_{2} \mathrm{H}_{5} \mathrm{O}-\stackrel{\mathrm{O}}{\mathrm{C}}-\underset{\mathrm{O}}{\mathrm{C}}{ }^{*} \mathrm{H}-\mathrm{O}_{\mathrm{O}}^{\mathrm{C}}{ }^{\star} \mathrm{H}-\stackrel{\mathrm{O}}{\mathrm{C}}-\mathrm{OC}_{2} \mathrm{H}_{5}+\mathrm{Br}-\left(\mathrm{CH}_{2}\right)_{6}-\mathrm{O}-\mathrm{N}=\mathrm{N}-\mathrm{NO}_{2} \longrightarrow \text { LCTT } \\
\mathrm{LCTT}+\mathrm{HO}-\left(\mathrm{CH}_{2}\right)_{4}-\mathrm{OH} \longrightarrow \text { LCPTT }
\end{gathered}
$$

Figure 10. Synthetic route of LCPTT.

of the mesogenic-chiral terminal group in ferroelectric liquid-crystalline polyacrylates, polymethacrylates and polysiloxanes. This restriction is effective for stabilizing the alignment of the polarization in LCPTT, and gives rise to macroscopic polarization. Consequently, $P_{s}$ of LCPTT is independent of the position of chiral center, and LCPTT exhibits large $P_{\mathrm{s}}{ }^{8,14}$

\section{EXPERIMENTAL}

\section{Measurements}

Molecular weight of polymer was determined by a Toso HLC-802UR GPC in THF, and calibrated against polystyrene standards.

Phase transition behavior was measured with DSC and a polarizing microscope equipped a Mettler FP52 hot stage with a Mettler FP5 temperature controller.

X-Ray diffraction was measured with a Rigaku X-ray Rad 2B system.

Birefringene was determined by an Abbe refractometer equipped a temperature controller. In this case, the polymer sample was oriented by rubbing treatment of the prism of the Abbe refractometer.

The dielectric constant was obtained with a YHP 4284A LCR meter. The measuring temperature was controlled with a Mettler FP52-FP5 system.

$P_{\text {s }}$ was estimated from the hysteresis loop observed by the Sawyer-Tower method.

Dielectric measurement and observation of hysteresis loop were carried out for a planarly aligned sample sandwiched between conductive coating glass plates. In this case, the electric field was applied between substrates.

\section{Synthesis}

The synthetic route of LCPTT is shown in Figure $10 .^{7,8} \mathrm{~A}$ solution of optically active diethyltartrate $(1.15 \mathrm{ml}, 6.7 \mathrm{mmol})$ in acetone $(50 \mathrm{ml})$ was added dropwise to a mixture of $p$-bromohexyloxy- $p^{\prime}$-nitroazobenzene $(5.5 \mathrm{~g}$, $13.5 \mathrm{mmol})$, tetra- $n$-butylammonium bromide (TBAB) $(4.4 \mathrm{~g}, 13.6 \mathrm{mmol})$, potassium carbonate $(1.9 \mathrm{~g}, 13.8 \mathrm{mmol})$ and acetone $(100 \mathrm{~mol})$, and the mixture was refluxed for $72 \mathrm{~h}$. The reaction mixture was filtered off, and the filtrate evaporated. The pure chiral tartrate (LCTT) was obtained by recrystallization from a mixed solution of chloroform and hexane. Yield: $66.3 \%,[\alpha]_{\mathrm{D}}^{25}+4.8^{\circ}\left(c 1, \mathrm{CHCl}_{3}\right)$. The products were characterized by IR and ${ }^{1} \mathrm{H}$ NMR spectroscopy.

LCPTT was prepared by the melt polycondensation of LCTT with 1,4-butanediol. A mixed solution of tetraisopropyl ortho titanate and diethylene glycol dimethyl ether was used as the polymerization catalyst. The reaction mixture was heated to melt $(110$ $120^{\circ} \mathrm{C}$ ) under dry nitrogen atmosphere. Then a few drops of above catalyst were added, and the reaction mixture was heated for $7 \mathrm{~h}$. Subsequently, the reaction temperature was raised at $140^{\circ} \mathrm{C}$, and atmospheric pressure was controlled at low vacuum $(0.026 \mathrm{~atm})$ for $3 \mathrm{~h}$. Finally, the reaction mixture was heated at $160^{\circ} \mathrm{C}$ under high vacuum $(0.0026 \mathrm{~atm})$ for $2 \mathrm{~h}$. After the reaction, the cooled reaction mixture was dissolved in tetrahydrofuran (THF), and LCPTT was reprecipitated by adding methanol to its THF solution.

\section{REFERENCES}

1. V. P. Shibaev, M. V. Kozlovsky, L. A. Beresnev, L. 
M. Blinov, and N. A. Plate, Polym. Bull., 12, 299 (1984).

2. G. Decobert, F. Soyer, and J. C. Dubois, Polym. Bull., 14, 179 (1985).

3. S. Uchida, K. Morita, K. Miyoshi, K. Hashimoto, and K. Kawasaki, Mol. Cryst. Liq. Cryst., 155, 93 (1988).

4. T. Suzuki, T. Okawa, T. Ohnuma, and Y. Sakon, Makromol. Chem., Papid Commun., 9, 755 (1988).

5. G. Scherowsky, A. Schliwa, J. Springer, K. Kuhnpast, and W. Trapp, Liq. Cryst., 5, 1281 (1989).

6. M. Dumon, H. T. Nguyen, M. Mauzac, C. Destrade, M. F. Achard, and H. Gasparoux, Macromolecules, 23, 355 (1990).
7. S. Ujiie and K. Iimura, Chem. Lett., 2217 (1989).

8. S. Ujiie and K. Iimura, Chem. Lett., 1031 (1990).

9. S. G. Kostromin, R. V. Talroze, V. P. Shibaev, and N. A. Plate, Makromol. Chem., Papid Commun., 3, 803 (1982).

10. H. Stevens, G. Rehage, and H. Finkelmann, Macromolecules, 17, 851 (1984).

11. F. Hessel and H. Finkelmann, Polym. Bull., 15, 349 (1986).

12. K. Sato, K. Otsuka, and M. Matsumoto, Makromol. Chem. Rapid Commun., 9, 631 (1988).

13. J. Hablutzel, Helv. Phys. Acta, 12, 489 (1939).

14. B. Otterholm, M. Nilsson, S. Lagerwall, and K. Skarp, Liq. Cryst., 2, 757 (1987). 